University of Nebraska - Lincoln

DigitalCommons@University of Nebraska - Lincoln

Nutrition and Health Sciences -- Faculty

Publications

Nutrition and Health Sciences, Department of

2007

\title{
Development and pilot testing of an omega-3 fatty acid food frequency questionnaire
}

\author{
Paula K. Ritter-Gooder \\ University of Nebraska-Lincoln, pgooder@alltel.net \\ Nancy M. Lewis \\ University of Nebraska--Lincoln, nlewis2@unl.edu \\ Kimberly Barber-Heidal \\ East Carolina University \\ Megan Waltz-Hill \\ University of Nebraska-Lincoln
}

Follow this and additional works at: https://digitalcommons.unl.edu/nutritionfacpub

Part of the Dietetics and Clinical Nutrition Commons

Ritter-Gooder, Paula K.; Lewis, Nancy M.; Barber-Heidal, Kimberly; and Waltz-Hill, Megan, "Development and pilot testing of an omega-3 fatty acid food frequency questionnaire" (2007). Nutrition and Health Sciences -- Faculty Publications. 11.

https://digitalcommons.unl.edu/nutritionfacpub/11

This Article is brought to you for free and open access by the Nutrition and Health Sciences, Department of at DigitalCommons@University of Nebraska - Lincoln. It has been accepted for inclusion in Nutrition and Health Sciences -- Faculty Publications by an authorized administrator of DigitalCommons@University of Nebraska - Lincoln. 
Published in Journal of Food Composition and Analysis 21, Supplement 1 (February 2008; 30th US National Nutrient Databank Conference), pp. S43-S49; doi: 10.1016/j.jfca.2007.05.003 Copyright @ 2007 Elsevier Inc. Used by permission.

Submitted November 14, 2006; revised April 5, 2007; accepted May 15, 2007; published online June 26, 2007.

\title{
Development and pilot testing of an omega-3 fatty acid food frequency questionnaire
}

\author{
Paula K. Ritter-Gooder, ${ }^{1}$ Nancy M. Lewis, ${ }^{1}$ Kimberly Barber-Heidal, ${ }^{2}$ and Megan Waltz-Hill ${ }^{1}$ \\ 1. University of Nebraska-Lincoln, Lincoln, NE 68588, USA \\ 2. East Carolina University, East Fifth St, Greenville, NC 27858-4353, USA \\ Corresponding author - P. K. Ritter-Gooder, tel 501 663-3550, fax 501 954-8882, email pgooder@alltel.net
}

\begin{abstract}
Omega-3 fatty acids are essential to health and provide cardio-protective benefits. To estimate omega-3 fatty acid dietary intake, we developed a semi-quantitative food frequency questionnaire (FFQ) and pilot tested its reliability and validity in a convenience sample of cardiac patients in the Midwest. The FFQ was developed using the USDA Nutrient Database for Standard Reference and the ESHA Food Processor, ESHA Research, Salem, Oregon. Three 24-h recalls and two administrations of the FFQ two weeks apart were obtained by a dietitian by phone and home visit from 28 volunteers. Pearson correlation was used to assess the association between food recalls and FFQ. Alpha coefficient was used to assess reliability of the FFQ. The correlation of omega-3 fatty acids intake using the food recalls and FFQ was $0.42(\mathrm{P}<0.05)$. Reliability of the FFQ was 0.83 with the food categories ranging from 0.93 for grains to 0.57 for vegetables. Ninety percent of omega- 3 fatty acid intake was provided by 28 foods, with the top two food sources from plant origin. The FFQ yielded excellent reliability and acceptable validity in the population tested and can be used to estimate total omega-3 fatty acid intake from plant, animal, and seafood sources in the diets of cardiac patients.
\end{abstract}

Keywords: omega-3 fatty acids, food frequency questionnaire, FFQ

\section{Introduction}

Omega-3 fatty acids are polyunsaturated fatty acids (PUFA) essential for health. They include alpha-linolenic acid (ALA), and the longer chain acids, eicosapentaenoic acid (EPA) and docosahexaenoic acid (DHA). The structural membrane lipids of nerve and retina tissue contain omega-3 fatty acids. Main dietary sources of ALA are nuts, seeds, and vegetable oils. Fish is the main concentrated dietary source of EPA and DHA. In addition, ALA can be converted to EPA and DHA in the body with varied conversion rates reported (Burdge, 2004). In 2002, dietary reference intakes for Adequate Intakes (AI) for omega-3 fatty acids was released by the National Academy of Sciences Institute of Medicine. The AI for ALA is $1.6 \mathrm{~g} /$ day for men aged 14 years to over 70 years and $1.1 \mathrm{~g} / \mathrm{d}$ for women of the same age group. The Acceptable Macronutrient Distribution Range (AMDR) for ALA is set at $0.6-1.2 \%$ of energy. Approxi- mately $10 \%$ of the total AMDR can be consumed as EPA and DHA (Institute of Medicine, 2002).

Research has been reported examining the relationship of omega-3 fatty acids to cardiovascular health and disease outcomes. Many of these studies evaluated fish and fish oil consumption by use of a food frequency questionnaire (FFQ) (Yuan et al., 2001; Siscovick et al., 2000; Ascherio et al., 1995). Other studies evaluated omega-3 fatty acid consumption, along with other nutrients, using a FFQ (Folsom and Demissie, 2004; Hu et al., 1999, 2002; Iso et al., 2001; Andersen et al., 1996; Pietinen et al., 1997).

Several studies have assessed fish and total omega-3 fatty acid intakes in the Midwestern population. Mean omega-3 fatty acid intakes in the diets of pregnant women $(1.060 \pm 0.300 \mathrm{~g} / \mathrm{d})$ (Lewis et al., 1995b), individuals with hypercholesterolemia $(0.620 \pm 0.710 \mathrm{~g} / \mathrm{d})$ (Lewis et al., 2000), and exercising individuals (0.887 $\pm 0.121 \mathrm{~g} / \mathrm{d}$ ) (Sindelar et al., 2004) were below the AI. 
A random telephone survey of households in the Midwest regarding the types and cuts of meat consumed revealed that fish/seafood was chosen infrequently approximately $10 \%$ of the time for noon and evening meals (Lewis et al., 1995a).

Due to the infrequent consumption of fish in Midwestern populations, assessment of omega-3 fatty acid intakes needs to include all food sources of omega-3 fatty acids. Consumption of large quantities of foods that provide low concentrations of omega-3 fatty acids can make important contributions to the diet. A FFQ to estimate intake from plant, animal, and seafood sources of omega-3 fatty acids is needed. Our goal was to develop and pilot test a FFQ to measure total omega-3 fatty acid dietary intake. Food recalls were compared to the FFQ to assess validity of the instrument in a group of volunteers with heart disease. To establish reliability of the FFQ, the results of two administrations of the FFQ were compared.

\section{Materials and methods}

A semi-quantitative FFQ design (Figure 1) was selected using small, medium, and large portions. The standard for medium portion size was defined by the USDA Food Guide Pyramid portions (USDA, 1992). A small serving is $1 / 2$ of a portion, a medium serving is one portion, and a large serving is $1 \frac{1}{2}$ portions. For meat, poultry, and seafood, a medium portion size was listed as $3 \mathrm{oz}$. Food items were classified into nine categories, includ- ing seafood, meats, eggs, milk/milk products, vegetables, fruits, grain products, fats/oils, and legumes. The eight frequency responses were none, once/month, <once/ week, 1-2 times/week, 3-4 times/week, 5-6 times/week, daily, and >once/day. The USDA National Nutrient Database for Standard Reference, Release 15 (USDA, 2002), and ESHA Food Processor (ESHA Research, 2003) were used to identify foods containing $\geq 10 \mathrm{mg}$ omega- 3 fatty acid/medium serving. Instructions for using the FFQ included a printed guide showing the fist, palm, hand, and fingers for estimating portion sizes.

During the development phase, the FFQ was used in two groups to test for material reliability and ease of use. Twenty-five college students in an introductory nutrition course completed the FFQ on two separate administrations that were a month apart (Waltz-Hill, 2002). Fifty-nine low income consumers, aged 21-73 years, completed the FFQ in a three week computerbased nutrition education program pilot study (Flanagan, 2000). These tests indicated that the FFQ was appropriate for use by consumers within a wide age range and economic levels.

The FFQ was adapted for use with cardiac patients (Heidal et al., 2004). Herbs/spices, nuts/seeds and other foods used by this population were added. Content validity was tested with a focus group of peer professionals. Each professional timed their own completion of the FFQ and answered general questions. To improve clarity, minor changes in the wording of the instructions and the portion size guide were made after this testing.

Food Frequency Questionnaire

Date

This form asks about your usual dietary intake over the past month. It takes about 20-30 minutes to complete. Please use the following instruction.

1. Read each food item. If you have not eaten this food in the past month, mark "none" and move onto the next food item.

2. Indicate whether you think your usual serving size is small $(S)$, medium $(M)$, or large $(L)$ by marking the correct serving size box. Please refer to the handout on "Portion Size: A Handy Guide" for a visual reference on medium serving sizes.

NOTE: A small (S) serving is equal to half $(1 / 2)$ the medium serving.

A medium (M) is equal to the medium servings listed on the form.

A large $(L)$ is equal to one and a half $\left(1^{1 / 2}\right)$ times as much or more of the medium serving.

3. Think over the past month. How often do you usually eat each of the following food items? Again, mark the box under the correct heading. Answer each question as best you can; estimate if you are not sure.

\begin{tabular}{|c|c|c|c|c|c|c|c|c|c|c|c|c|}
\hline $\begin{array}{l}\text { Seafood \& } \\
\text { Fish }\end{array}$ & $\begin{array}{l}\text { Medium } \\
\text { Serving }\end{array}$ & None & $\mathrm{S}$ & $\mathbf{M}$ & $\mathbf{L}$ & $\begin{array}{l}\text { Once a } \\
\text { month }\end{array}$ & $\begin{array}{l}\text { Less than } \\
\text { once a week }\end{array}$ & $\begin{array}{l}\text { 1-2 times } \\
\text { a week }\end{array}$ & $\begin{array}{l}\text { 3-4 times } \\
\text { a week }\end{array}$ & $\begin{array}{l}\text { 5-6 times } \\
\text { a week }\end{array}$ & Daily & $\begin{array}{l}\text { More than } \\
\text { once a day }\end{array}$ \\
\hline Tuna & 3 ounces & & & & & & & & & & & \\
\hline Salmon & 3 ounces & & & & & & & & & & & \\
\hline Whitefish & 3 ounces & & & & & & & & & & & \\
\hline Herring & 3 ounces & & & & & & & & & & & \\
\hline Walleye & 3 ounces & & & & & & & & & & & \\
\hline Lake trout & 3 ounces & & & & & & & & & & & \\
\hline
\end{tabular}

Figure 1. n-3 Fatty acid food frequency questionnaire sample page. 
The instrument was then used in a group of cardiac subjects $(n=36)$ to survey dietary omega- 3 fatty acid intake and food selections as part of a nutrition study.

A pilot study was conducted to validate the 152 item FFQ against omega-3 fatty acid intake estimates using food recalls (Ritter-Gooder et al., 2006). The instrument was tested in a convenience group of volunteers, 17 women and 11 men who were involved in the earlier nutrition study. Volunteers were invited by phone to participate in this study. Volunteers were informed that the purpose of the study was to evaluate the usefulness of the FFQ as a method to measure dietary intake of omega-3 fatty acids. They were not advised that the FFQ would be compared to the food recalls. All components of the study were approved by the Institutional Review Board.

At a home visit, a dietitian collected a 24-h recall and administered the FFQ. The food recall was obtained using an interview similar to the USDA 5-Step Multiple Pass Method (Conway et al., 2003). Volunteers were queried in five steps. Step one collected a quick list of foods and beverages consumed. In step two, an inquiry was made into categories of foods that have been documented as frequently forgotten, such as crackers with soup or milk with cereal. In step three, the time and occasion when the foods were consumed were obtained. A description of foods and the amounts eaten was gathered in step 4. Step 5 provided the final probe into completeness of the food recall. Food models, measuring utensils, and printed visuals were used for portion size estimations. After the recall, volunteers were asked to fill out the FFQ. They were instructed to respond by recalling the frequency of consumption of each of the foods listed within the past month. Volunteers were asked to record two additional 24-h food recalls within the next week. They were asked not to use sequential days and to include 1 weekend day. These two food recalls were collected by telephone. To measure reliability of the instrument, participants completed the second FFQ again two weeks later and returned the questionnaire by self-addressed stamped envelope provided.

ESHA Food Processor (ESHA Research, 2003) was used to analyze the food recalls and mean daily consumption was determined. On the FFQ, the intake of omega-3 fatty acids was calculated by multiplying the amount of omega- 3 fatty acids for each food item by the frequency and serving size selected. Omega-3 fatty acid intakes and estimates of daily omega-3 fatty acid intake by food group were calculated using Microsoft@ Excel (2002, Microsoft Corporation). Data were analyzed using Statistical Package for the Social Sciences (version 11.0, 2002, SPSS. Inc., Chicago, IL). Pearson correlation was used to assess the association between food recalls and FFQ using log transformed data. Alpha coefficient (Fleiss, 1986) was used to assess reliability of the FFQ and food categories within the tool.

\section{Results}

All 28 volunteers enrolled completed the study. Over $60 \%$ of the volunteers were female. Nearly $90 \%$ were between the ages of 43-69, with the remaining between 70 and 80 years of age (Table 1). The correlation of omega- 3 fatty acid intakes using the food recalls and FFQ was 0.42 $(P<0.05)$. The difference in daily omega-3 fatty acid intake between the FFQ and three 24-h recalls was .705 g \pm 0.393 (SE). A paired sample $t$ test indicated no significant difference between the two assessment methods $(P \geq$ $0.05)$. Reliability of the FFQ was 0.83 with the food groupings ranging from 0.93 for grain products to 0.57 for vegetables (Table 2). The rank order of food categories contributing to omega-3 fatty acid intake show nuts/seeds, and fats/oils as the first two categories, with seafood as the third category (Table 3). Twenty eight foods contributed $90 \%$ of omega-3 fatty acid intake. The individual food rankings reveal the top two foods as English walnuts and flax seed (Table 4). A total of 73 out of 152 foods contributed $98 \%$ of the omega- 3 fatty acid intake. Overall 113 out of 152 items on the FFQ were consumed. Average time to complete the FFQ was $20 \mathrm{~min}$.

\section{Discussion}

The FFQs used in studies on cardiovascular disease and omega-3 fatty acids have varied in content and purpose. Questionnaire design, classification of foods and

Table 1. Sociodemographic characteristics of volunteers with heart disease $(n=28)$ who completed 3 food recalls and 2 FFQs

\begin{tabular}{lr}
\hline Characteristics & $n(\%)$ \\
\hline Sex & \\
Male & $11(39)$ \\
Female & $17(61)$ \\
Age $(y)$ & \\
$43-59$ & $13(46)$ \\
$60-69$ & $12(43)$ \\
$70-80$ & $3(11)$ \\
Education $(y)$ & \\
$\leq 12$ & $8(29)$ \\
$13-15$ & $3(11)$ \\
16 or more & $11(39)$ \\
Other & $6(21)$ \\
Marital Status & \\
Married & $19(68)$ \\
Single, never married & $1(3)$ \\
Widowed & $3(11)$ \\
Divorced or separated & $5(18)$ \\
Income a & \\
$\leq 24,999$ & $6(22)$ \\
25,000-34,999 & $4(14)$ \\
35,000-59,999 & $12(43)$ \\
$\geq 60,000$ & $5(18)$ \\
\hline
\end{tabular}

a. In US\$. One response is missing for the data presented. 
Table 2. Reliability coefficients of categories of foods providing omega- 3 fatty acids ${ }^{\mathrm{a}}$ in volunteers with heart disease $(n=28)$

\begin{tabular}{lc}
\hline Food categories & Alpha coefficient \\
\hline Grain products & 0.93 \\
Fats/oils & 0.86 \\
Seafood & 0.84 \\
Legumes & 0.82 \\
Nuts/seeds & 0.81 \\
Meats & 0.76 \\
Herbs/spices & 0.73 \\
Fruits & 0.68 \\
Eggs & 0.65 \\
Milk/milk products & 0.59 \\
Vegetables & 0.57 \\
\hline
\end{tabular}

a. Intake assessed by food frequency questionnaire.

Table 3. Food category contributions to total daily omega-3 fatty acid intakes ${ }^{\mathrm{a}}$ consumed by volunteers with heart disease $(n=28)$

\begin{tabular}{llr}
\hline Rank order & Food category & \% omega-3 fatty acid intake \\
\hline 1 & Nuts/seeds & 59.30 \\
2 & Fats/oils & 6.80 \\
3 & Seafood & 5.60 \\
4 & Legumes & 5.25 \\
5 & Grain products & 4.50 \\
6 & Meats & 3.02 \\
7 & Fruits & 1.78 \\
8 & Vegetables & 1.78 \\
9 & Eggs & 1.20 \\
10 & Milk/milk products & 0.69 \\
\hline
\end{tabular}

a. Intake assessed by food frequency questionnaire.

number of food items were based on the purpose of the study and population studied. Some FFQs have been designed for use in estimating EPA and DHA, which are primarily in seafood. These FFQs vary in classification of fish and number of food items. Yuan et al. (2001) used a 45 item FFQ that grouped seafood into three categories, fresh, salted, and shellfish to observe the relationship of fish and shellfish consumption to death from myocardial infarction in Chinese men. A 78 item FFQ grouping seafood into three categories, mixed Mediterranean fish, canned fish, and other fish such as octopus and squid, was used by Tavani et al. (2001) to study the relationship between omega- 3 fatty acids, fish, and nonfatal myocardial infarction in Italian women and men. Four seafood items were included in the 131 item FFQ that Ascherio et al. (1995) used in evaluating dietary intake of marine omega-3 fatty acids and fish with risk of coronary disease among men in the Health Professional Follow-up Study. Siscovick et al. (2001) estimated long chain omega-3 PUFA intakes with a FFQ that listed 25 fish and 10 shellfish in Pacific Northwest subjects.

Other FFQs have been used to estimate total omega-3 fatty acids, among other nutrients. In the Nurses Health Study Cohort, Hu et al. (1999) measured dietary ALA and the risk of fatal ischemic heart disease. The FFQ consisted of 116 items and nine responses of frequencies and was designed to assess the intake of specific fats and other nutrients. This same FFQ was used in studying the association of fish and omega- 3 fatty acids intake to women's stroke risk by Iso et al. (2001). In more recent studies of omega-3 fatty acids and coronary heart disease, Hu et al. (2002) and Folsom and Demissie (2004) used a similar version of the same FFQ.

A FFQ for measuring dietary seafood intake is described by Wood et al. (2002). Ten frequencies, ranging from less than once per month to three or more times per day, were measured in the instrument which collected macro- and micronutrient intakes. Both frequency of fish consumption and the preparation method were collected in the study of young Australian adults. Dietary intake of fatty acids in Norwegians was measured by Andersen et al. (1996) using a 180 item quantitative FFQ. A larger 276 item FFQ, completed by Finnish men for estimating intake of fatty acids, was used in a study of the risk of coronary heart disease by Pietinen et al. (1997).

In contrast to the previously developed FFQs, our objective was to develop a FFQ that exclusively estimated total dietary omega-3 fatty acid intake. The FFQ was designed to collect dietary intake of omega-3 fatty acids from all food sources that contained at least $10 \mathrm{mg}$ omega-3 fatty acid per medium serving. The frequency questionnaire included 152 foods and 26 of these were seafood items that were available in the Midwestern food supply.

Average correlations between food recalls and FFQ are in the range of $r=0.4-0.6$ (Block and Hartman, 1989; Willet, 1998; Thompson and Byers, 1994) for a variety of nutrients. Our correlation of 0.42 is within this range. Lower correlations occur for Vitamin E (0.19) and Vitamin A (0.41). Huang et al. (2002) reported the correlations for phystoestrogens contained in soy products as $0.49-0.58$ for daidzein and $0.45-0.54$ for genistein. All these dietary components are similar to omega- 3 fatty acids in that they occur in high concentrations in a few foods and in low concentrations in the majority of foods.

Results of the pilot testing of the FFQ in volunteers indicated the reliability of the FFQ was high (alpha coefficient $=0.83$ ). The closer the alpha coefficient is to 1.00 the greater the internal consistency of items in the instrument being assessed. This demonstrates that each volunteer was able to estimate and give the same information on intake from one administration of the questionnaire to the next administration two weeks later. Our alpha coefficient was higher than that measured by Erkkola et al. (2001) in a Finnish population (0.62) when assessing omega-3 fatty acids by FFQ. Morris et al. (2003) assessed Vitamin E intakes using FFQs in Chicago Health and Aging Project participants and reported a coefficient of 0.67 . Our results indicate that alpha coefficients varied among food categories. The differences suggest the volunteers had more difficulty 
Table 4. Rank order listing of foods that provided $90 \%$ of daily n-3 fatty acid intake ${ }^{\text {a }}$ of volunteers with heart disease $(n=28)$

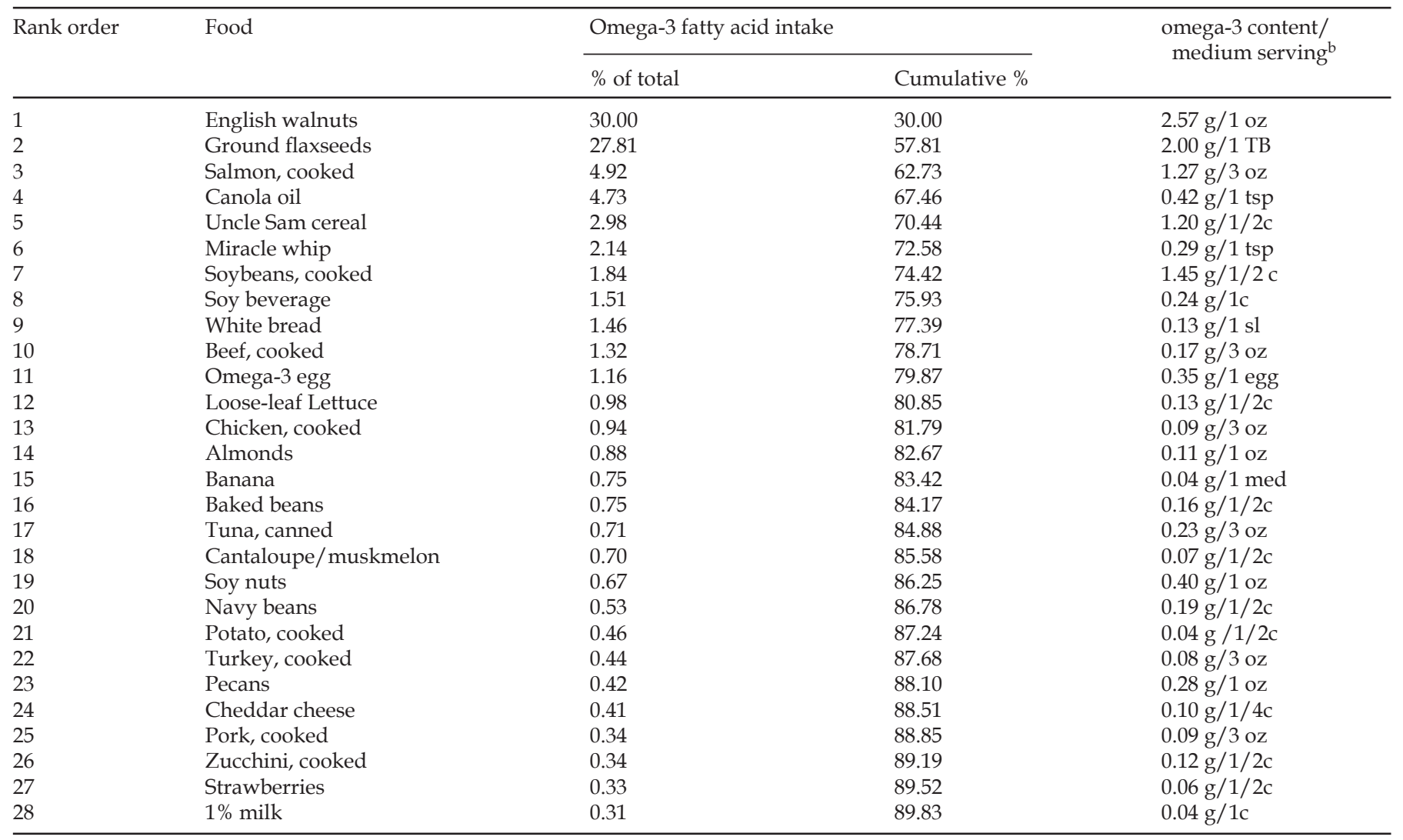

a. Intake assessed by food frequency questionnaire.

b. Values obtained from Food Processor, Version 8.1, 2003, ESHA Research, Salem, OR.

remembering intakes of some foods than others. Milk/ milk products and vegetables had the lowest coefficients indicating these are subject to more variation than grain products and fats/oils which had higher correlations. No food category fell below 0.57 .

Collecting multiple dietary recalls provides the most accurate estimates of usual intakes. Due to the highly variable amounts of omega-3 fatty acids among foods, three 24-hour recalls may not reflect usual intake. Nes et al. (1992) validated a FFQ used to estimate dietary intake of fatty acids by collecting 14-day weighed diet records. For estimating fat intake, Basiotis et al. (1987) reported that 54 days for males and 71 days for females is required to get within $\pm 10 \%$ accuracy with a $95 \%$ confidence level. However, we chose three 24-h recalls to lower respondent burden. The schedule of the FFQ administrations and the food recalls was for the convenience of the volunteers as they had participated in the earlier study. Their previous experience in completing the FFQ may have increased the reliability measurement of the instrument due to familiarity of use. The administration of the first FFQ occurred immediately after the 24-h food recall during the home visit. While collecting a food recall just prior to completing the FFQ may have affected the response to the FFQ, we found high reliability upon second administration of the FFQ two weeks later. Volunteers were asked about their frequency of consumption within the past month. We decided to collect the second FFQ within this time frame to avoid recording actual changes in food selections that may occur over a longer time period.

Nuts/seeds were the top food source of omega-3 fatty acids in this population and provided nearly $60 \%$ of the intake. Our findings suggest that these volunteers may not consume fish or sufficient quantity of fish to obtain adequate dietary amounts of omega-3 fatty acids. The ability to assess diets for total omega- 3 fatty acid intake from all dietary sources is therefore important.

This FFQ could be developed further by including estimation of ALA, EPA, and DHA subtotals. This will provide additional information and make it possible to distinguish intakes of different types of omega-3 fatty acids. Less than one-half of the food items listed on the FFQ contributed close to $100 \%$ of the estimated intake. Therefore it is likely that the number of food items on the FFQ can be reduced while still maintaining accuracy of assessment. Foods, such as infant formula and whole milk, will need to be included when using the tool with other populations, such as infants and children. The addition of mixed food such as pizza should be added to the FFQ. The availability of omega-3 enriched processed foods has been shown to increase population intakes 
of the nutrient in low fish eaters by Patch et al. (2005). Omega- 3 fatty acids are being incorporated into foods due to advances in technology (Whelan and Rust, 2006). As omega-3 fatty acid fortified foods become available, the FFQ should be updated. This FFQ could also be further validated using serum biomarkers.

FFQs are typically used to capture usual and long term dietary intake, can be completed independently by subjects after brief instruction on use, and are relatively inexpensive to administer. While the FFQ has a lower respondent burden than recalls, less specificity is obtained by use of a FFQ and its use in clinical practice is limited. Correspondingly, 24-h recalls and food records are time consuming and resource intensive. Clinicians may need a brief tool to quickly estimate intakes for use with patients and to measure eating behavior changes for outcomes documentation.

\section{Conclusions}

The FFQ yielded excellent reliability and acceptable validity in the population tested. This FFQ can be used to estimate omega-3 fatty acid intakes but needs more testing due to the small number of subjects studied. For use in future studies, the number of food items can be reduced to lower respondent burden. When used with other groups or in different regions, additional foods consumed by that population may need to be added to the FFQ. In future studies, it may be necessary to estimate ALA, EPA, and DHA intake in certain populations. Further modification of the tool can provide these estimates.

\section{References}

Ascherio et al., 1995 • A. Ascherio, E. B. Rimm, M. J. Stampfer, E. L. Giovannucci, and W. C. Willet, Dietary intake of marine $n-3$ fatty acids, fish intake, and the risk of coronary disease among men, The New England Journal of Medicine 332 (1995), pp. 977-982.

Andersen, 1996 - L. F. Andersen, K. Solvodl, and C. A. Drevon, Very-long-chain $n-3$ fatty acids as biomarkers for intake of fish and $n-3$ fatty acid concentrates, American Journal of Clinical Nutrition 64 (1996), pp. 305-311.

Basiotis et al., 1987 - P. P. Basiotis, S. O. Welsh, F. J. Cronin, J. L. Kelsay, and W. Mertz, Number of days of food intakes records required to estimate individual and group nutrient intakes with defined confidence, Journal of Nutrition 117 (1987), pp. 1638-1641.

Block and Hartman, 1989 - G. Block and A. M. Hartman, Issues of reproducibility and validity of dietary studies, American Journal of Clinical Nutrition 50 (1989), pp. 1133-1138.

Burdge, 2004 - G. Burdge, Alpha-linolenic acid metabolism in men and women: Nutritional and biological implications, Current Opinion Clinical Nutrition Metabolism Care 7 (2004), pp. 137-144.
Conway et al., 2003 - J. Conway, L. Ingwersen, B. Vinyard, and A. Moshfegh, Effectiveness of the US Department of Agriculture 5-step multiple pass method in assessing food intake in obese and nonobese women, American Journal of Clinical Nutrition 77 (2003), pp. 1171-1178.

Erkkola et al., 2001 • M. Erkkola, M. Karppinen, J. Javarainen, L. Rasanen, M. Knip, and S. M. Virtanen, Validity and reproducibility of a food frequency questionnaire for pregnant Finnish women, American Journal of Epidemiology 151 (5) (2001), pp. 466-476.

ESHA Research, 2003 - ESHA Research. Food Processor, Version 8.1, 2003, Salem, OR: http://www.esha.com

Flanagan, 2000 - N. L. Flanagan, Evaluating the effects of multi media nutrition education on the low income consumer's consumption of omega-3 fatty acids and stage of change advancement for fat and fiber intake, Thesis, University of Nebraska-Lincoln, 2000.

Fleiss, 1986 - J. L. Fleiss, Design and Analysis of Clinical Experiments, Wiley, New York (1986).

Folsom and Demissie, 2004 - A. R. Folsom and Z. Demissie, Fish intake, marine omega-3 fatty acids, and mortality in a cohort of postmenopausal women, American Journal of Epidemiology 160 (2004), pp. 1005-1010.

Heidal et al., 2004 - K. Heidal, N. Lewis, and S. Evans, Survey of omega-3 fatty acid intakes and omega- 3 food selections in cardiac patients living in a section of the Midwestern United States, Nutrition Research 24 (2004), pp. 741-747.

Hu et al., 1999 • F. B. Hu, M. J. Stampfer, J. E. Manson, E. B. Rimm, A. Wolk, G. A. Colditz, C. H. Hennekens, and W. C. Willet, Dietary intake of a-linolenic acid and risk of fatal ischemic heart disease among women, American Journal of Clinical Nutrition 69 (5) (1999), pp. 890-895.

Hu et al., 2002 - F. B. Hu, W. Willet, M. J. Stampfer, K. M. Rexrode, C. M. Albert, D. Hunter, and J. E. Manson, Fish and Omega-3 fatty acid intake and risk of coronary heart disease in women, Journal of the American Medical Association 787 (2002), pp. 1815-1821.

Huang et al., 2002 • M. Huang, G. Harrison, M. Mohamed, J. Gornbein, S. Henning, V. Go, and F. Greendate, Assessing the accuracy of a food frequency questionnaire for estimating usual intake of phytoestrogens, Nutrition Cancer 37 (2002), pp. 145-155.

Institute of Medicine, 2002 - Institute of Medicine, 2002. Dietary Reference Intakes for Energy, Carbohydrates, Fiber, Fat, Protein and Amino Acids (Macronutrients). The National Academies Press, Washington, DC, p. 1325 (Dietary Reference Intakes).

Iso et al., 2001 - H. Iso, K. M. Rexrode, M. J. Stampfer, J. E. Manson, G. A. Colditz, F. E. Speizer, C. H. Hennekens, and W. C. Willett, Intake of fish and omega-3 fatty acids and risk of stroke in women, Journal of the American Association 285 (3) (2001), pp. 304-312.

Lewis et al., 1995a - N. M. Lewis, A. C. Widga, J. S. Buck, and A. M. Frederick, Survey of omega-3 fatty acids in diets of Midwest low-income pregnant women, Journal of Agromedicine 2 (1995), pp. 49-57.

Lewis et al., 1995b - N. M. Lewis, J. A. Albrecht, M. I. Schnepf, F. L. Hamouz, J. A. Driskell, and J. A. Goertz, Meat choices and cookery methods of Nebraskans, Journal Foodservice Systems 8 (1995), pp. 165-174. 
Lewis et al., 2000 - N. M. Lewis, K. Schlach, and S. E. Scheideler, Serum lipid responses to $n-3$ fatty acid enriched eggs in persons with hypercholesterolemia, Journal of the American Dietetic Association 100 (2000), pp. 365-367.

Morris et al., 2003 - M. C. Morris, C. C. Tangney, J. L. Bienias, D. A. Evans, and R. S. Wilson, Validity and reproducibility of a food frequency questionnaire by cognition in an older biracial sample, American Journal of Epidemiology 158 (12) (2003), pp. 1213-1217.

Nes et al., 1992 - M. Nes, A. L. Frost, and K. Solvoll et al., Accuracy of a quantitative food frequency questionnaire applied in elderly Norwegian women, European Journal of Clinical Nutrition 46 (1992), pp. 809-821.

Patch et al., 2005 - C. S. Patch, L. C. Tapsell, T. A. Mori, B. J. Meyer, K. J. Murphy, J. Mansour, M. Noakes, P. M. . Clifton, I. B. Puddey, L. J. Beilin, G. Annison, and P. R. Howe, The use of novel foods enriched with long-chain $n-3$ fatty acids to increase dietary intake: a comparison of methodologies assessing nutrient intake, Journal of the American Dietetic Association 105 (2005), pp. 1918-1926.

Pietinen et al., 1997 - P. Pietinen, A. Ascherio, P. Korhonen, A. M. Hartman, W. C. Willett, D. Albanes, and J. Virtamo, Intake of fatty acids and risk of coronary heart disease in a cohort of Finnish Men, American Journal of Epidemiology 145 (1997), pp. 876-887.

Ritter-Gooder et al., 2006 - P. K. Ritter-Gooder, N. M. Lewis, K. B. Heidal, and K. M. Eskridge, Validity and reliability of a quantitative food frequency questionnaire measuring n-3 fatty acid intakes in cardiac patients in the Midwest: A validation pilot study, Journal of the American Dietetic Association 106 (2006), pp. 1251-1255.

Sindelar et al., 2004 • C. Sindelar, S. Scheerger, S. Plugge, K. Eskridge, R. C. Wander, and N. M. Lewis, Serum lipids of physically active adults consuming omega-3 fatty acidenriched eggs or conventional eggs, Nutrition Research 24 (2004), pp. 731-739.

Siscovick et al., 2000 - D. S. Siscovick, T. E. Raghunathan, I. King, S. Weinmann, V. E. Bovbjerg, L. Kushi, L. A. Cobb, M. K. Copass, B. M. Psaty, R. Lemaitre, B. Retzlaff, and
R. H. Knopp, Dietary intake of long-chain $n-3$ polyunsaturated fatty acids and the risk of primary cardiac arrest, American Journal of Clinical Nutrition 71 (Suppl.) (2000), pp. 208S-212S.

Tavani et al., 2001 - A. Tavani, C. Pelucchi, E. Negri, M. Bertuzzi, and C. L. Vecchia, Polyunsaturated fatty acids, fish, and nonfatal acute myocardial infarction, Circulation 104 (2001), pp. 2269-2272.

Thompson and Byers, 1994 - F. E. Thompson and T. Byers, Dietary assessment resource manual, Journal of Nutrition 124 (Suppl 115) (1994), pp. 2245S-2317S.

Waltz-Hill, 2002 - M. Waltz-Hill, Using multimedia nutrition education to increase consumer understanding of coronary heart disease, Thesis, University of Nebraska-Lincoln, 2002.

Whelan and Rust, 2006 - J. Whelan and C. Rust, Innovative dietary sources of omega-3 fatty acids, Annual Review of Nutrition 26 (2006), pp. 75-103.

Willet, 1998 - W. Willet, Nutritional Epidemiology (2nd ed.), Oxford University Press, New York, NY (1998).

Wood et al., 2002 • R. K. Wood, R. M. Stoney, P. D. Ireland, M. J. Baileyu, J. M. Raven, F. C. K. Thien, E. H. Walters, and M. K. Abramson, A valid food frequency questionnaire for measuring dietary fish intake, Asia Pacific Journal of Clinical Nutrition 11 (2002), pp. 56-61.

Yuan et al., 2001 • J. Yuan, R. K. Ross, Y. Gao, and M. C. Yu, Fish and shellfish consumption in relation to death from myocardial infarction among men in Shanghai, China, American Journal of Epidemiology 154 (2001), pp. 809-816.

USDA, 2002 • US Department of Agriculture (USDA), Agricultural Research Service, 2002. USDA National Nutrient Database for Standard Reference, Release 15. Retrieved February 20, 2007, from the Nutrient Data Laboratory home page: http://www.ars.usda.gov/ba/bhnrc/ndl

USDA, 1992 • US Department of Agriculture (USDA), Human Nutrition Information Service, 1992. Food Guide Pyramid. A Guide to Daily Food Choices. Home and Garden Bulletin No. 252. 